\title{
Distribution network planning with a large amount of small scale photovoltaic power
}

\author{
Alberto Fernández Martínez \\ School of Electrical Engineering \\ Royal Institute of Technology \\ Stockholm, Sweden \\ albfer@kth.se
}

\author{
Yalin Huang \\ IEEE Student Member \\ School of Electrical Engineering \\ Royal Institute of Technology \\ Stockholm, Sweden
}

\author{
Lennart Söder \\ IEEE Member \\ School of Electrical Engineering \\ Royal Institute of Technology \\ Stockholm, Sweden
}

\begin{abstract}
This paper presents a new method to assist distribution system operators assessing the capacity of each bus for connecting more photovoltaic power in a low voltage distribution network. The developed method takes the uncertainties from load and generation into account. Moreover, it applies probabilistic voltage limits in the assessment process. An improved linear power flow analysis is used in the process. Furthermore, a case study in a Swedish low voltage network is discussed to apply the proposed method on assessing the maximum photovoltaic peak power that can be installed into a distribution network without violating probabilistic voltage limits. A comparison between deterministic approach and probabilistic approach is also performed.
\end{abstract}

Index Terms - distribution network, low voltage, planning, photovoltaic power, probabilistic

\section{INTRODUCTION}

Many national governments around the world are supporting the increase of small scale distributed generation (DG) connections by implementing different programs, especially related to roof mounted photovoltaic (PV) solar systems [1]. These connections mainly take place at low voltage (LV) level in distribution network (DN) [2]. The increasing penetration level of DG in deregulated electric power systems introduces more uncertainties to the distribution network planning process. The connection point of a DG unit is uncertain for distribution system operators (DSOs), instead of being centrally planned as in the traditional power systems. Therefore, it is important for DSOs to assess the capacity of each bus for connecting more DG in a LV network. This capacity is referred as hosting capacity. Moreover, the production from DG is stochastic. This increases the uncertainties in the power flow in the network. Applying deterministic limits in the worst-case scenario is not realistic [3]. The quality of power supply regulated by the European standard EN 50160 [4] allows voltage magnitude variations in a limited time. Therefore, the probabilistic planning criteria, e.g. not only the voltage level but also how often can these limits be violated, are recommended to be implemented in the planning process [5].

This paper presents the main results from a project which belongs to KIC InnoEnergy Sweden, Smart Power Action 2.3.
Load flow (LF) calculations are employed in network planning to ensure that voltage and current values are kept in accordance to the established regulations [1]. Traditionally, planning approaches have focused LF on extreme conditions, while the likelihood of these events is not considered [5]. Although probabilistic approaches have been used in power system analysis since the 70s [6], deterministic approaches have meanwhile still been employed by transmission system operators (TSOs) and DSOs due to their easy and fast results [7].

However, deterministic planning methodologies are not able to handle uncertainties [7]. These methodologies can lead to uneconomical or unreliable solutions [3]. For instance, results shown in [1] conclude that a deterministic load flow (DLF) overestimates the maximum PV peak power that can be installed in a radial DN with PV DG units. In addition, results presented in [8] support the need of considering these uncertainties in the calculations when choosing an optimal alternative when network planning. In the case study of a real system, an increase of $75 \%$ connected DG capacity is obtained when uncertainties are considered, in comparison to the case where uncertainties are disregarded [8].

While a deterministic approach solves a specific state of the system, usually the worst case conditions; a probabilistic assessment describes the more likely states of the system [7]. A probabilistic load flow (PLF) allows evaluating the probabilistic variation of voltages at all nodes in a grid [5]. In comparison to the worst case planning principle, statistical planning of DN increases the flexibility of the planning decisions based on an increase of the number of possible scenarios studied [9]. Consequently, a probabilistic methodology is needed to provide reliable voltage evaluations [5].

This paper develops a probabilistic method to assist DSOs to assess the hosting capacity of each bus in the network. The outputs of the methods are not only hosting capacity but also the weak bus that limits this capacity. It takes the stochastic behavior of load and PV into account and applies probabilistic planning criteria. In addition, the application of a linear power flow analysis makes the method easy to be implemented. 


\section{UNCERTAINTIES IN NETWORK PLANNING}

\section{A. Uncertainties from $P V$ and load}

Different models can be used to represent the uncertainty of load variation and solar radiation. One of them is to describe the uncertainty in terms of a probabilistic density function (PDF) [1]. The Normal distribution is widely used to describe the load variation at a bus in a DN. For instance, in $[1],[5],[9]$, and [10], the load is considered as a normally distributed random variable within each hour. The total load of the network is represented in [1] by a single probability density function assuming that all loads are fully correlated. The feeder load is then shared between nodes based on their contract rated capacity.

Moreover, loads can be categorized into groups. A customer group can be constructed by summing the similar individual customer load curves statistically [9]. The seasonal and daily behavior of the load demand due to human work and rest habits is shown in [11], which are presented in terms of mean value and standard deviation.

The power output of a PV panel is proportional to the irradiance on a surface with a given inclination [12]. Two different approaches to model a PV panel production are applied in [13]: the k-means clustering and the normal density probability function. When applying the normal distribution, deviations of the daily energy capacity of the PV panel is between $9 \%$ and 13\%. More detailed PV generation models are also found. A model based on the inclination, declination, reflectance of the ground, the latitude, etc. is presented in [1]. In [14] solar radiation in a time step is assumed to be dependent on the beam radiation, the diffuse radiation, the slope of the PV surface, the ground reflectance, the anisotropy and the cloudiness. In addition, the unavailability of the PV units is considered in [15].

\section{B. Probabilistic voltage limits: European standard EN 50160}

The quality of the voltage is the most relevant issue in LV network planning [1]. The European standard EN 50160 regulates the voltage characteristics in public distribution systems [16] . According to the standard EN 50160, the supply voltage should stay within the range of $\pm 10 \%$ of the nominal voltage during $95 \%$ of each period of one week, when measuring 10 minutes mean rms values [7], [16]. This restriction is presented in Table I:

TABLE I. EN 50160: SLOW VOLTAGE MAGNITUDE VARIATIONS IN VOLTAGE CHARACTERISTICS IN PUBLIC DISTRIBUTION SYSTEMS

\begin{tabular}{|c|c|c|c|c|}
\hline Parameter & $\begin{array}{c}\text { Acceptable } \\
\text { limits }\end{array}$ & $\begin{array}{c}\text { Measurement } \\
\text { interval }\end{array}$ & $\begin{array}{c}\text { Monitoring } \\
\text { period }\end{array}$ & $\begin{array}{c}\text { Acceptance } \\
\text { average }\end{array}$ \\
\hline $\begin{array}{c}\text { Slow } \\
\text { voltage } \\
\text { magnitude } \\
\text { variation }\end{array}$ & $\begin{array}{c}\text { Rated } \\
\text { voltage } \\
\pm 10 \%\end{array}$ & 10 minutes & 1 week & $95 \%$ \\
\hline
\end{tabular}

\section{METHODOLOGY}

The proposed method requires probabilistic inputs from load and PV production in order to apply a probabilistic voltage limit in determining the hosting capacity of a bus.
A simplified power flow which favors later calculation is developed. A PLF with Monte Carlo simulation permits to analyze all possible operation scenarios. The hosting capacity is further scaled to the peak power of a PV unit. This is logical because the PV panels in the market are mostly described by their peak capacities.

\section{A. Including load and PV uncertainties}

Uncertainty derived from load and generation is usually tackled by scenario technique [17]. Scenarios of DG production as well as load are divided into summer and winter, weekday and weekend, and 24 hours a day in [11]. In the proposed method, hourly scenarios are used. Each PV production and load consumption in an hour is characterized by a PDF. Monte Carlo simulation is used to sample data in a scenario.

\section{B. Linear power flow analysis in LV network}

The single line equivalent in Fig. 1 presents a four-bus radial system. The series impedance between bus $i$ and bus $j$, denoted as $\underline{Z}_{i j}$, consists of a resistance $R_{i j}$ and a reactance $X_{i j}$. Additionally, a power $S_{3}$ is injected to bus 3 .

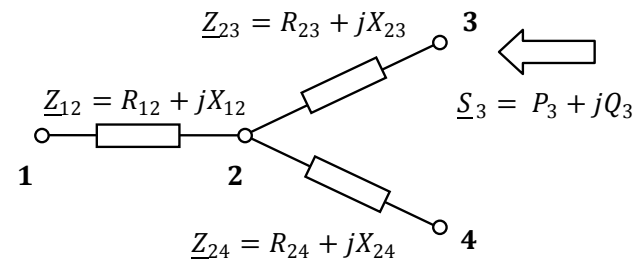

Figure 1. Single power injection in a bus of a radial LV network

It is assumed that the angle shift between voltages is very small. The voltage difference between bus 3 and bus 1 is, at $\underline{S}_{4}=0$, calculated using (1).

$U_{3}-U_{1}=\frac{\left(R_{12}+R_{23}\right) \cdot P_{3}+\left(X_{12}+X_{23}\right) \cdot Q_{3}}{U_{3}}$

Assuming bus 1 is the slack bus $\left(U_{1}=1\right.$ p. u. $)$, the voltage variation on bus 3 can be calculated by (3). A new variable $\Delta W$ is introduced to represent the Impact of Squared Voltage (ISV) from a power injection at any bus in the network in (4). $\Delta W_{3}^{3}$ is the ISV at bus 3 due to the power injection $\underline{S}_{3}$. There is a direct relation between voltage variation and ISV, such as (5).

$$
\begin{gathered}
\Delta U_{3}=U_{3}-1 \\
\Delta U_{3}=\frac{\left(R_{12}+R_{23}\right) \cdot P_{3}+\left(X_{12}+X_{23}\right) \cdot Q_{3}}{1+\Delta U_{3}} \\
\Delta W_{3}^{3}=\left(R_{12}+R_{23}\right) \cdot P_{3}+\left(X_{12}+X_{23}\right) \cdot Q_{3} \\
\Delta W_{3}^{3}=\Delta U_{3} \cdot 1+\left(\Delta U_{3}\right)^{2}
\end{gathered}
$$

The ISV at bus 4 due to power $\underline{S}_{3}$ is only dependent on $\underline{Z}_{12}$ :

$$
\Delta W_{4}^{3}=R_{12} \cdot P_{3}+X_{12} \cdot Q_{3}
$$


For any two buses in a radial network:

$$
\Delta W_{t}^{i}=R(i, t) \cdot P_{i}+X(i, t) \cdot Q_{i}
$$

where $\Delta W_{t}^{i}$ denotes the ISV at bus $t$ due to the power injected to bus $i$. The variables $R(i, t)$ and $X(i, t)$ represent the resistance and reactance of the common line of bus $i$ path and bus $t$ path respectively. Path is used to describe the line between a bus and the slack bus in this paper. $P_{i}$ and $Q_{i}$ refer to the active and reactive power injected to bus $i$ respectively. Note that the variable $P_{i}$ represents the difference between the active power $P g_{i}$ generated and the active load $P l_{i}$ demanded in the given bus $i$. The same applies to $Q_{i}$. Therefore, at net demand, the injection is negative.

Any new connection affects the bus voltage at all buses in the network. The ISV of each bus contains the impacts from all power injections in the network on that bus, as $\sum_{i} \Delta W_{t}^{i}$ in (8). Therefore, the capacity of squared voltage variation on bus $k$ limited by bus $t$ is given by (8):

$$
\Delta W_{t}^{k}=\Delta W_{\text {lim }}(t)-\sum_{i} \Delta W_{t}^{i}, \quad t \in \Omega_{m}, i \in \Omega_{n}
$$

where $\Delta W_{\text {lim }}(t)$ denotes the limit of ISV on bus $t . \Delta W_{t}^{k}$ shows the maximum ISV allowed on bus $t$ for the new connection on bus $k$. $\Omega_{m}$ represents a set containing all buses in the network; while $\Omega_{n}$ represents a set containing only the buses where the power injection is not zero but bus $k$.

Integrating (7) into (8), the hosting capacity of bus $k$ limited by bus $t$ can be calculated by (9):

$$
P_{k}(t)=\frac{\left(\Delta W_{\text {lim }}(t)-Q_{k} \cdot X(k, t)-\sum_{i}\left[P_{i} \cdot R(i, t)+Q_{i} \cdot X(i, t)\right]\right)}{R(k, t)}
$$

$$
t, k \in \Omega_{m}, i \in \Omega_{n}
$$

where bus $k$ denotes the connection bus of a new PV unit. $P_{k}(t)$ denotes the hosting capacity of bus $k$ limited by the voltage variation on bus $t$. Each bus has a certain capacity of ISV left, $\Delta W_{t}^{k}$, for the new connection. Moreover, the location of the new connection represented in $R(k, t)$ and $X(k, t)$ affects the hosting capacity. Therefore, it is not enough to calculate the hosting capacity of a bus just based on the lowest ISV capacity $\left(\Delta W_{t}^{k}\right.$ in (8)). It is necessary to assume each bus as the limiting bus to calculate $P_{k}(t)$. The minimum among all the $P_{k}(t)$ values is the permissible new power injection in the existing network. The bus that causes the minimum $P_{k}$ is referred to as the weak bus, which limits the hosting capacity of bus $k$.

\section{Planning according to probabilistic limits}

The proposed method is able to obtain the hosting peak power of a PV unit in a LV distribution network, as well as the weak bus that limits the connection according to probabilistic voltage limits. The European standard EN 50160 is used as an example to assist explanation, but the method is not limited to only apply this standard. Fig. 2 shows a flowchart of the proposed method.

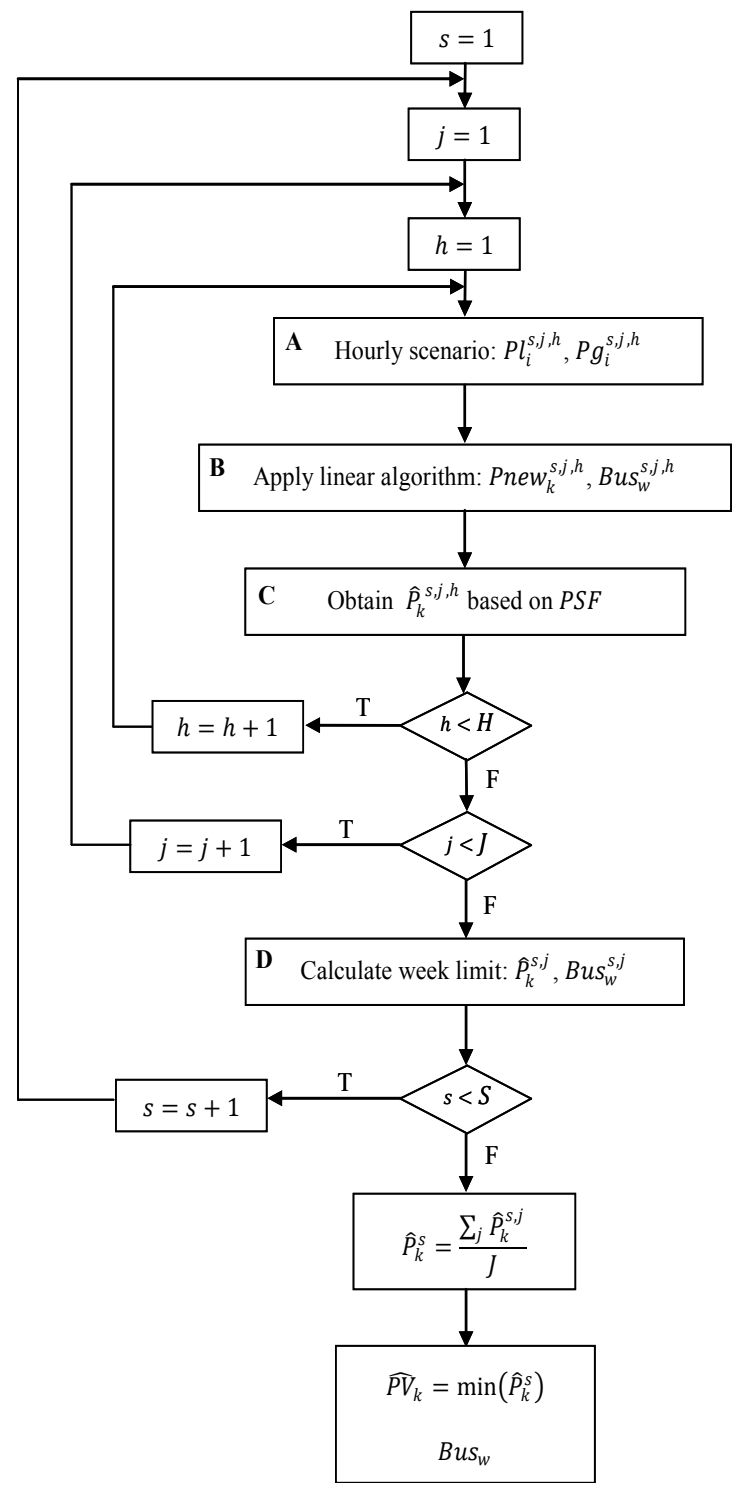

Figure 2 Flowchart of the proposed method

For each specific period of a year $(s=1, \ldots, S)$, a total number of weeks $(j=1, \ldots, J)$ are solved on an hourly $(h=1, \ldots, H)$ basis following the next steps:

1) Create an hourly scenario. Load consumption $\left(P l_{i}^{s, j, h}\right.$ with $\left.i=1, \ldots, n_{T}\right)$ and generation $\left(P g_{i}^{s, j, h}\right)$ of existing $\mathrm{PV}$ units are randomized corresponding to hourly PDFs respectively. (Box A in Fig. 2)

2) Analyze the power flow by the proposed linear process in Section II-B. The hosting capacity of bus $k$ during this hour $\left(\right.$ Pnew $\left._{k}^{s, j, h}\right)$ is calculated, as well as the limiting bus $\left(B u s_{w}^{s, j, h}\right)$ by (9). (Box B in Fig. 2)

3) Scale the hosting capacity to the hosting peak power of a PV unit. (Box C in Fig. 2) 
The production scaling factor (PSF) is used in this step. The PSF of a PV unit during one hour is defined as the ratio between the mean PV production and the peak production of the PV unit. It is assumed to be known during any hour at a certain location. The maximum hosting peak power of the new PV unit $\left(\hat{P}_{k}^{s, j, h}\right)$ is converted from the hosting capacity of bus $k$. PSF is zero when there is no sunlight, therefore:

$$
\begin{aligned}
& \text { a) If } P S F=0 \text {, then } \hat{P}_{k}^{s, j, h}=0 . \\
& \text { b) If } P S F \neq 0 \text {, then } \hat{P}_{k}^{s, j, h}=P n e w_{k}^{s, j, h} / P S F
\end{aligned}
$$

4) Repeat Steps 1) to 3) until the counter $h$ equals the value $H(H=168$ according to EN 50160).

5) Repeat Step 4) until the counter $j$ equals the value $J$.

6) Select the maximum peak power of the new PV unit for each week and corresponding weak bus in each scenario $\left(\hat{P}_{k}^{s, j}\right.$, Bus $\left._{w}^{s, j}\right)$. (Box D in Fig. 2)

This step incorporates a function that selects the maximum power of the new PV unit in each week by identifying a bus, in which the number of voltage violations first reaches 8 hours (5\% of a week), according to EN 50160. Hourly allowed maximum power injections in a week are ordered from minimum to maximum. Then, being the first implies being more likely to cause over-voltage problems with the lowest values of peak power installed by the PV unit.

7) Repeat Steps 1) to 6) a total of $S$ times, i.e. a whole year is considered. By convergence of all values obtained in step 6) for each period $s$, the power limit of the new PV unit $\left(\hat{P}_{k}^{s}\right)$ is obtained. The minimum value among them is the peak power of the new PV unit that can be hosted on bus $k$ :

$$
\widehat{P V}_{k}=\min \left(\hat{P}_{k}^{s}\right) \quad \forall s \in\{1, \ldots, S\}
$$

\section{Validation of the proposed planning method}

Newton Raphson power flow is used in the validation simulation, instead of the linear power flow analysis. Since Newton Raphson power flow requires power injections as input, the hosting capacity of a bus is calculated by an iterative simulation. The peak power of the new PV is increased step by step until the probability of the voltage violation limit is violated. This probability is represented by the maximum number of hours the voltage violation is allowed $\left(H_{\text {lim }}\right)$. The result obtained by the proposed method is the start value of this iterative simulation. It follows the next steps:

1) Create an hourly scenario. Load consumption $\left(P l_{i}^{s, j, h}\right)$ and generation $\left(P g_{i}^{s, j, h}\right)$ of existing PV units are sampled following their corresponding hourly PDFs. The production of the new PV unit is also sampled based on its assumed peak power $\left(\operatorname{Pnew}_{k}^{s, j, h}\right)$.
2) Analyze the load flow in the network using the NewtonRaphson algorithm. The voltage at every bus is obtained $\left(V_{i}^{s, j, h}\right)$.

3) Count the number of voltage violations. The voltage level of every bus is compared to the voltage limit $V_{\text {max }}$. If it is over the limit, counter $N B_{i}^{s, j}$ adds one.

4) Repeat steps 1) to 3) until the counter $h$ reaches the value $H$. The variable $N B_{i}^{s, j}$ represents the total number of hours a bus $i$ breaks its over-voltage limit during a week $j$ of the periods.

5) Repeat step 4) until the counter $j$ equals the value $J$.

Steps 1) to 5) are then repeated a total of $S$ times, i.e. for all periods in a year. Variable $N B_{i}^{s, j}$ is obtained for every season $s$ and every simulated week $j$ for each bus $i$. By convergence of all values obtained for each bus $i$ during each period $s$, the average number of hours each bus $i$ breaks during a week of each season $s$ is obtained $\left(N B_{i}^{S}\right)$. The weak bus is the one breaking the maximum number of hours during a week:

$$
N B_{\text {weak }}=\max \left(N B_{i}^{S}\right) \quad \forall s \in\{1, \ldots, S\} \text { and } i \in \Omega_{n}
$$

In the case that $N B_{\text {weak }}$ is below the value $H_{\text {lim }}$, the peak power of the new PV unit is increased by a defined iterative step and the complete process is repeated again. Otherwise, if $N B_{\text {weak }}$ has a higher value than $H_{\text {lim }}$, the iterative process ends. Consequently, the peak power of the new permissible PV unit corresponds to the value employed in the iteration previous to the latest one. The weak bus in the network due to this new connection is also obtained.

\section{CASE STUDY}

A Swedish LV network with high potential for PV connections is studied. All calculations are implemented in MATLAB. According to the Swedish electrical legislation Ellag (1997:857), DSOs have to maintain a good quality of the transfer of electricity [18]. There is no uniform definition regarding the voltage levels that Swedish DSOs must apply for planning [19]. However, the voltage limits presented in the European standard EN 50160 are included in a Swedish regulation called EIFS 2011:2, which was published by "Energy Markets Inspectorate" in June 2011 [19].

\section{A. LV distribution network}

Fig. 3 represents a single-phase equivalent circuit of the three-phase Swedish LV network. The nominal three-phase voltage of the network is $400 \mathrm{~V}$ and it consists of a total of 18 buses. The network is compounded of five main feeders. Load points are located at the end of the feeders. The slack bus is set as the LV busbar of the feed-in transformer. The feed-in transformer is a $10 / 0.4 \mathrm{kV}$ transformer with a rated apparent power of $200 \mathrm{kVA}$. More detailed description of this network can be found in [20]. The planning problem is to determine the maximum allowed peak power of a PV unit to be connected to Bus 10 in this network, where five PV units are already installed. The PV units already installed in buses 02, 07, 08, 09 and 17 have a peak power of $40 \mathrm{~kW}, 32 \mathrm{~kW}, 35 \mathrm{~kW}, 12$ $\mathrm{kW}$ and $20 \mathrm{~kW}$ respectively. 


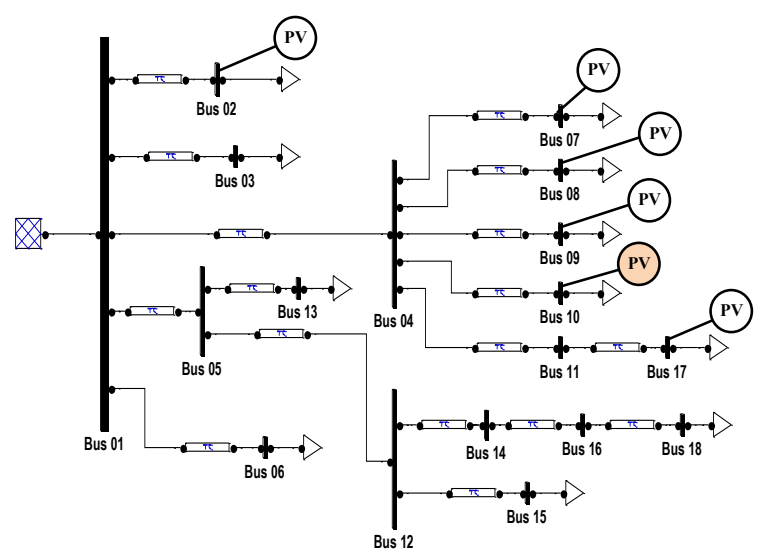

Figure 3. Planning problem: a new PV connection to Bus 10 with five existing PV units

\section{B. Load consumption model}

According to the consumption behavior in Sweden, the characteristics of hourly electrical loads are divided into three seasons (summer, autumn and winter), weekday and weekend. Consequently a total of $144(3 \times 2 \times 24)$ representative hours are used for each kind of load. Two different load groups are considered: residential and industrial. All loads are assumed to be normally distributed in each hour and all loads in the same group are fully correlated.

In each hour, the mean value is calculated by:

$$
\text { Mean value }(\mathrm{kW})=L S F \cdot \text { Nominal value }(\mathrm{kW})
$$

The LSF is defined as the ratio between the mean load consumption and the nominal power of the load. The LSF varies in every hour for each group. LSF of both a residential and an industrial load during the 24 hours of a weekday in spring are considered as base case. These values are obtained from [21], where a 24 hour load characteristic for a thirty-bus IEEE system is depicted. Values of LSF for weekends and other seasons are derived from this base case by application of correction factors. Correction factors are chosen according to [22] and [23].

For each hour, the standard deviation is calculated by:

\section{Standard deviation $(\mathrm{kW})=R S D \cdot$ Mean value $(\mathrm{kW})$}

The relative standard deviation (RSD) of a normal distribution is defined as the ratio between the standard deviation and the mean value. The RSD is different for residential and industrial loads. In the case study presented in this paper, a value of $10 \%$ and $5 \%$ is used for residential and industrial loads respectively. These values have been chosen following [1] and [5].

\section{Photovoltaic generation model}

Power produced by each PV unit is assumed to be normal distributed every hour of the year. Following the Swedish standard SvKFS 2005:2, PV units are assumed to produce with a constant power factor one [24]. The seasonal variation of PV arrays power output in Sweden has been shown by a test carried out by Energy Agency [25]. According to that, daily and seasonal behavior of the PV power output is considered by dividing the power production into three seasons (summer, autumn and winter) and in 24 hours a day.
Consequently a total of $72(3 \times 24)$ representative hours are used. PV units are assumed to be available all year long.

The solar radiation in every node of the network is assumed to be fully correlated. The same probability distribution is then used for every PV connected to the network. In each hour, the mean value of the normal distribution is calculated based on the PSF and the peak power. PSF variation of a real PV unit during the 24 hours of a weekday in summer is considered as base case. These values are obtained from available production data of a PV panel of $1 \mathrm{kWp}$ peak power. These data belong to the department of Ingeniería Eléctrica in E.T.S.I Industriales de la Universidad Politécnica de Madrid. PSF for winter and spring are $30 \%$ and $70 \%$ of the base case. In addition, according to hours of sunlight, there are more zero values of PSF for winter. In this case study, a value of 5\% is used as RSD for all PV units.

\section{Results}

The over-voltage limit established by the European standard EN 50160 is applied into the proposed method, and the worst-case scenario is also analyzed in the case study. The permissible peak power of a new PV unit at Bus 10 is obtained $35.11 \mathrm{~kW}$ and $14.09 \mathrm{~kW}$ respectively. In the validation method a value of $35.56 \mathrm{~kW}$ is obtained. A considerable improvement is observed in the result when applying the proposed method and it is very close to the validation value.

In Fig. 4 and Fig. 5, the 168 voltage values on Bus 10 obtained during each hour of a summer week have been plotted for each method. Bus 10 is identified as the weak bus in this case study. According to the standard EN 50160, the over-voltage limit of 1.1 p.u., which is highlighted with a red vertical line in both figures, can be violated a maximum of 8 hours per week. The result of the proposed method is slightly smaller than the reference value, since the over-voltage limit is broken just 7 hours.

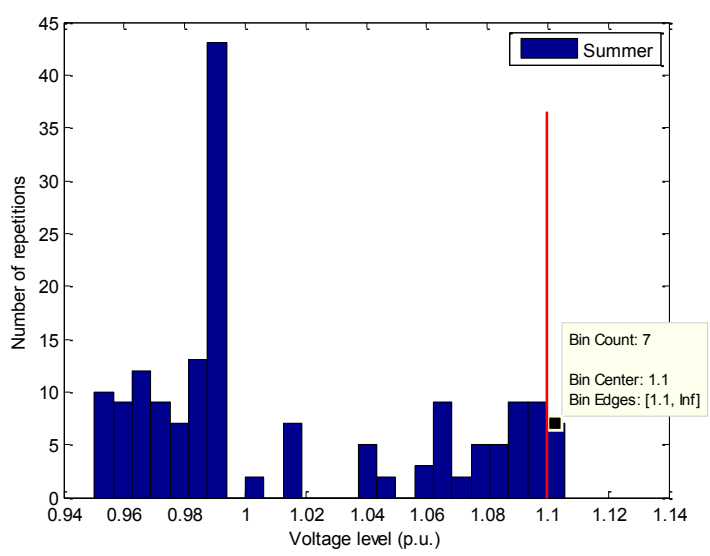

Figure 4. Voltage on Bus 10 with a PV unit of $35.11 \mathrm{~kW}$

The result shows that summer conditions are the worst ones from an over-voltage perspective in this case study. In this network, load consumption is lower during summer than 
during winter or spring. Additionally, the PV production is higher during summer. Therefore, a summer week is characterized by low demand and high PV production. Reverse power flow is favored and voltage levels are expected to rise, increasing the risk of breaking the over-voltage limit established by the standard EN 50160.

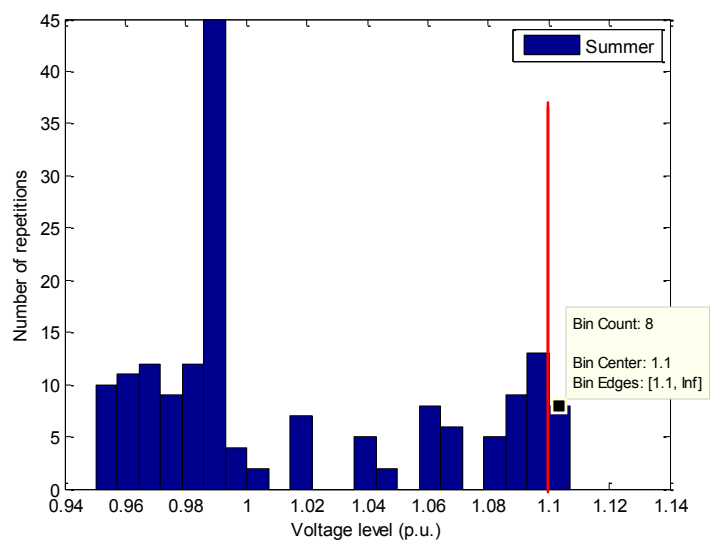

Figure 5. Voltage on Bus 10 with a PV unit of $35.56 \mathrm{~kW}$

\section{CONCLUSION}

This paper presents a probabilistic assessment method for LV distribution networks with a high penetration of intermittent generation such as PV. The proposed method is able to assess the hosting capacity of a bus in LV networks considering the uncertainties from PV generation and load. Probabilistic voltage limits established by EN 50160 are embedded in the method; however, it is flexible enough to change to other probabilistic standards on voltage variation. A linear power flow analysis is developed to assist the assessment. It results in the capacity of any bus in a network for hosting a new PV connection and the weak bus in the network that limits the connection. Therefore, this method can be used as a general guideline for the DSO to assess and recommend connection points for $\mathrm{PV}$ generators in $\mathrm{LV}$ networks.

\section{REFERENCES}

[1] S. Conti och S. Raiti, "Probabilistic load flow using Monte Carlo techniques for distribution networks with photovoltaic generators," Solar Energy, vol. 81, nr 12, pp. 1473-1481, 2007.

[2] P. Richardson och A. Keane, "Impact of high penetrations of microgeneration on low voltage," i 20th International Conference and Exhibition on Electricity, Prague, 2009.

[3] G. Celli och F. Pilo, "MV Network Planning under uncertainties on Distributed Generation penetration," i Power Engineering Society Summer Meeting, Vancouver, 2001.

[4] E. 50160, "Voltage characteristics of electricity supplied by public distribution systems," 1999.

[5] S. Chun-Lien, "Stochastic Evaluation of Voltages in Distribution Networks with Distributed Generation Using Detailed Distribution Operation Models," IEEE Transactions on Power Systems, vol. 25, nr 2, pp. 786-795, 2010.

[6] D. Villanueva, A. Feijóo och J. Pazos, "Probabilistic Load Flow Considering Correlation between Generation, Loads and Wind Power," Smart Grid and Renewable Energy, vol. 2, nr 1, pp. 12-20, 2011.
[7] R. Villafáfila, B. Bak-Jensen och S. Sörensen, "Probabilistic assessment of wind power production on voltage profile in distribution network," i 9th International Conference in Electrical Power Quality and Utilisation, Barcelona, 2007.

[8] C. Tancredo Borges och V. Ferreira Martins, "Multistage expansion planning for active distribution networks under demand and Distributed Generation uncertainties," Electrical Power and Energy Systems, vol. 36, nr 1, pp. 107-116, 2012.

[9] S. Repo, H. Laaksonen och P. Järventausta, "Statistical models of distributed generation for distribution network planning," i 18th International Conference on Electricity Distribution, Turin, 2005.

[10] N. Hatziargyriou, T. Karakatsanis och M. Papadopoulos, "Probabilistic load flow in disribution systems containing dispersed wind power generation," IEEE Transactions on Power Systems, vol. 8, nr 1, pp. 159-165, 1993.

[11] P. Chen, Z. Chen, B. Bak-Jensen, R. Villafáfila och S. Sörensen, "Study of Power Fluctuation from Dispersed Generations and loads and its impact on a Distribution Network through a probabilistic approach," i 9th International Conference in Electrical Power Quality and Utilisation, Barcelona, 2007.

[12] B. Kroposki, K. Emery, D. Myers och L. Mrig, "A comparison of photovoltaic module performance evaluation methodologies for energy ratings," i 1st WCPEC, Hawaii, 1994.

[13] A. Di Piazza, M. Di Piazza och G. Vitale, "Statistical Processing of Data Coming from a Photovoltaic Plant for Accurate Energy Planning," i International Conference on Renewable Energy and Power Quality, Santander, 2008.

[14] J. Zou, K. Zhou, K. Lei och Z. Zhang, "A Method to Optimize the Placement of PV-Wind-Storage Hybrid System," i Power and Energy Engineering Conference, Asia-Pacific, Shanghai, 2012.

[15] V. Mendez, J. Rivier, J. de la Fuente och T. Gómez, ”A Monte Carlo approach for assessment of investments deferral in radial distribution networks with distributed generation," i IEEE Bologna PowerTech Conference, Bologna, 2003.

[16] H. Markiewicz och A. Klajn, "Voltage Disturbances. Standard EN50160 - Voltage Characteristics in Public Distribution Systems," Wroclaw University of Technology, Poland, July 2004.

[17] A.O.Buygi,H.M.Shanechi,G.Balzer,E.Shahidehpour,N.Pariz, "Network planning in unbundled power systems," IEEE Transactions on power systems, vol. 21, nr 3, pp. 1379-1387, 2006.

[18] J. Lindhal, "National Survey Report of PV Power Applications in Sweden 2011," Swedish Energy Agency, Uppsala, May 2012.

[19] L. Ström, M. H. J. Bollen och R. Kolessar, "Voltage quality regulation in Sweden," i CIRED 21st International Conference on Electricity Distribution, Frankfurt, June 2011.

[20] A. Fernández Martínez, "Grid planning with a large amount of small scale solar and wind power," MS thesis, KTH.DiVA, Stockholm, 2013.

[21] W. El-Khattam, Y. Hegazy och M. Salama, "Investigating Distributed Generation Systems Performance Using Monte Carlo Simulation," IEEE Transactions on power systems, vol. 21, nr 2, pp. 524-532, 2006.

[22] M. Eisenreich, G. Balzer, J. Backes och B. Maurer, "Integration of PV systems into low voltage networks using standard load profiles," i International Conference on Renewable Energies and Power Quality (ICREPQ'10), Granada, 2010.

[23] R. Li, C. Gu, Y. Zhang och F. Li, "Implementation of load profile test for electricity distribution networks," i Power and Energy Society General Meeting, San Diego, 2012.

[24] "Anslutning av mindre produktionsanläggningar till elnätet - AMP," Svensk Energi - Swedenergy - AB, Sweden, November 2011.

[25] "Energimyndigheten," [Online]. Available: http://energimyndigheten.se/sv/Hushall/Testerresultat/Testresultat/Sol cellssystem-/?tab=1. [Använd 27 May 2013]. 